\title{
Particle Swarm Optimization Applied to EEG Source Localization of Somatosensory Evoked Potentials
}

\author{
Yazdan Shirvany, Student Member, IEEE, Qaiser Mahmood, Student Member, IEEE, Fredrik Edelvik, \\ Stefan Jakobsson, Anders Hedström, and Mikael Persson, Member, IEEE
}

\begin{abstract}
One of the most important steps in presurgical diagnosis of medically intractable epilepsy is to find the precise location of the epileptogenic foci. Electroencephalography (EEG) is a noninvasive tool commonly used at epilepsy surgery centers for presurgical diagnosis. In this paper, a modified particle swarm optimization (MPSO) method is used to solve the EEG source localization problem. The method is applied to noninvasive EEG recording of somatosensory evoked potentials (SEPs) for a healthy subject. A 1 mm hexahedra finite element volume conductor model of the subject's head was generated using $\mathrm{T} 1$-weighted magnetic resonance imaging data. Special consideration was made to accurately model the skull and cerebrospinal fluid. An exhaustive search pattern and the MPSO method were then applied to the peak of the averaged SEP data and both identified the same region of the somatosensory cortex as the location of the SEP source. A clinical expert independently identified the expected source location, further corroborating the source analysis methods. The MPSO converged to the global minima with significantly lower computational complexity compared to the exhaustive search method that required almost 3700 times more evaluations.
\end{abstract}

Index Terms-Electroencephalogram (EEG) source localization, finite element method (FEM), inverse problem, magnetic resonance imaging (MRI), particle swarm optimization, somatosensory evoked potential (SEP), subtraction method.

\section{INTRODUCTION}

$\mathbf{E}$ PILEPSY is one of the most common neurologic diseases in the world. According to the Centers for Disease Control and Prevention [1], epilepsy affects 2.2 million Americans. The Institute of Medicine, in their recent report "Epilepsy Across the Spectrum" [2] says "the 2.2 million prevalence estimate is most accurately viewed as approximating a midpoint in a wide potential range of 1.3 million to 2.8 million people with epilepsy." Epilepsy affects 65 million people worldwide. Many patients with epilepsy never receive the treatment they need in order to be seizure free; consequently, treatment of epilepsy with medications is a major effort of the World Health Organization [3].

Manuscript received January 15, 2013; revised June 30, 2013; accepted August 07, 2013. Date of publication October 07, 2013; date of current version January 06, 2014.

Y. Shirvany, Q. Mahmood, and M. Persson are with the Department of Signals and Systems, Chalmers University of Technology and MedTechWest Center, 41276 Göteborg, Sweden (e-mail: yazdan.shirvany@chalmers.se).

F. Edelvik and S. Jakobsson are with the Fraunhofer-Chalmers Research Centre, Chalmers Science Park, SE-412 88 Göteborg, Sweden.

A. Hedström is with the Sahlgrenska Academy, 41345 Göteborg, Sweden.

Color versions of one or more of the figures in this paper are available online at http://ieeexplore.ieee.org.

Digital Object Identifier 10.1109/TNSRE.2013.2281435
However, in cases of medically intractable epilepsy, surgical therapy is often the only feasible intervention.

The combination of the neuronal network approaches open up a short remark for an assumption that uncontrolled network oscillations in epilepsy need a cortical focus for high frequency activity during seizures. The spikes, the epileptiform activity in the interval between seizures, also need cortical foci [4]. Thus, source localization of epileptic activity is a tool to delineate cortical areas/volumes with abnormal neuronal activity of cells and networks. A correct and anatomically precise localization of the epileptic focus is mandatory for deciding whether resection of brain tissue is possible.

An electroencephalogram (EEG) is the most commonly used clinical method for finding the sources of brain activity. EEG source localization deals with two problems: 1) the forward problem in which scalp potentials are simulated for a (set of) given current source(s) in the brain, 2) the inverse problem in which a (set of) source(s) in the brain are estimated that fit with a given potential distribution at the scalp electrodes.

In the past, simplified spherical head models were used to solve the forward problem. More recently, a combination of imaging modalities (e.g., T1-,T2/PD- MR and DTI, [5]-[7]) are used to accurately describe the geometry of the head and conductivity of tissues. A finite element method (FEM) is well suited to handle the geometrical complexity of realistic head models and tissue inhomogeneity. The EEG source localization is (usually) highly nonlinear and requires efficient algorithms for its solution. Parametric methods based on current dipoles are well suited for estimating locally activated neural sources, e.g., events like epileptic spikes or motor control tasks [8], [9]. In these methods, a search is made for the best-fit dipole position(s) and orientation(s) and the methods assume a fixed number of dipoles inside the brain volume during the searching process. The most widely used parametric optimization methods for solving the EEG inverse problem can be classified into two groups: gradient methods, which use function and derivative information (e.g., Levenberg-Marquardt [10]), and search methods (nongradient techniques) which use only function values (e.g., Nelder-Mead downhill simplex [11]). Both of these methods minimize the cost function by iteratively adjusting the parameters of the dipole sources. The gradient optimization methods are fast to converge and effective when there is only one dipole in our source model and the data is noiseless. But when we use the multi-dipole model and noisy cases the local optimization approaches are no longer effective since it is easy to be trapped in local minima [12]-[14]. Moreover, the final solution often depends on the 


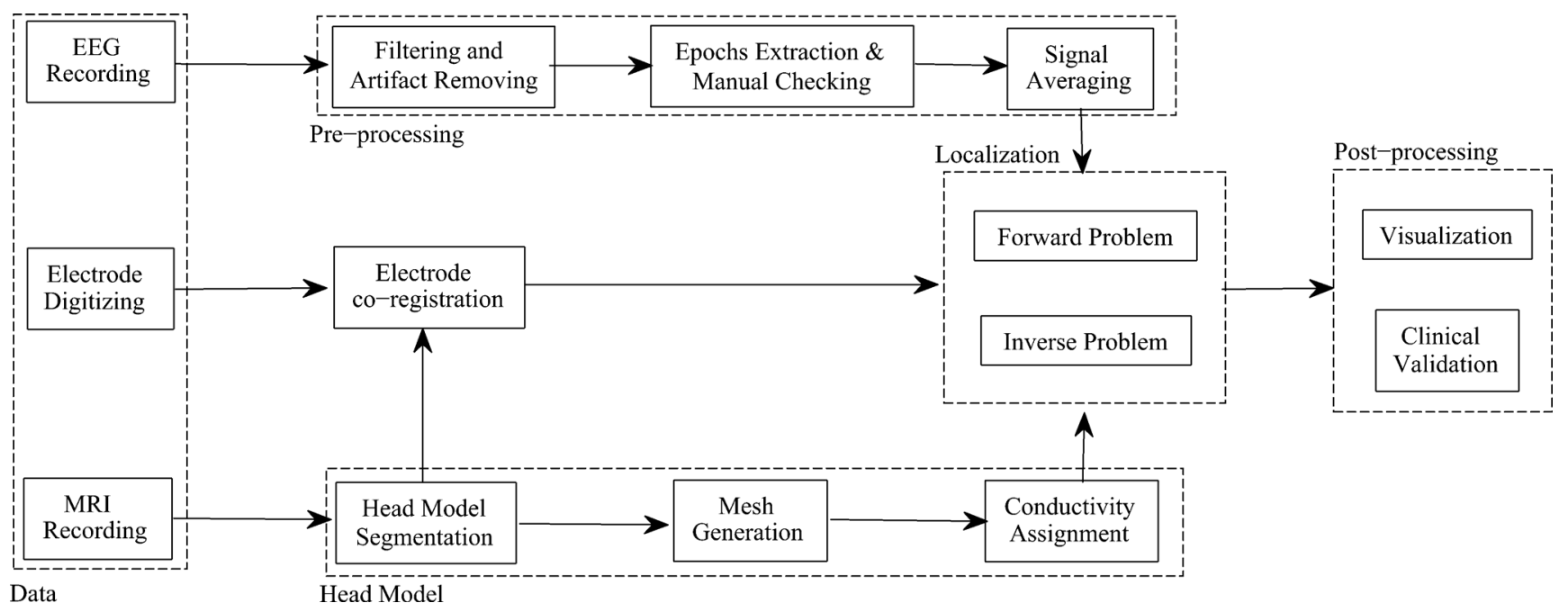

Fig. 1. Different steps in the EEG source localization procedure.

initial approximation and the number of local minima of the cost function [15] since reasonable initial guesses are difficult to make.

In previous studies done by the authors, [16], [17], a modified particle swarm optimization (MPSO) method was proposed for solving the EEG source localization. In [16], it is shown in several examples that, where as the deterministic method DIvide RECTangle (DIRECT) failed to efficently solve the source localization problems, the MPSO could found the optimal solution significantly faster than other improved versions of PSO, as well as GA. In addition, the MPSO is less prone to be trapped in local minima. Comparing the convergence rate between MPSO and GA in [16] showed that the MPSO converged to the global minima in all cases whereas the mean of the GA's convergence was $79 \%$. In [17], it is shown that the MPSO is feasible to solve the EEG source localization in a real clinical setup.

The main goal of this paper is to apply the MPSO method on real EEG data and compare the results with physiological knowledge. The real EEG data that we use in the present study was recorded from somatosensory evoked potentials (SEPs) stimulation on a healthy subject. The rest of the paper is organized as follows. Section II-A introduces the forward problem and a modified version of the subtraction method used to model the dipole source. Section II-B describes the inverse problem and its mathematical formulation. Section III presents the PSO, its parameter selection and modification methods. Section IV deals with the EEG data and the head model. The results are presented in Section V. Finally, Section VI discusses the results and conclusions are drawn.

\section{METHOD}

The EEG source localization has several different subproblems that each should be done carefully. Fig. 1 illustrates all necessary steps performed in the EEG source localization procedure. In the following sections we go through each step and present our proposed method for solving the EEG source localization.

\section{A. Forward Problem}

The characteristic frequencies of the signals in the kilohertz range and below make the capacitive and inductive effects of the tissue negligible. Therefore, the quasi-static approximation of Maxwell's equations for the potential $\Phi$ can be used. If we denote the domain of interest as $\Omega$ (with boundary $\partial \Omega$ ) and let the tissue conductivity be $\sigma$, we have Poisson's equation

$$
\nabla \cdot(\sigma \nabla \Phi)=\nabla \cdot \mathbf{j}^{s} \text { in } \Omega
$$

subject to the conditions

$$
\begin{aligned}
\hat{\mathbf{n}} \cdot(\sigma \nabla \Phi) & =0 \text { on } \partial \Omega \\
\Phi\left(\mathbf{x}_{\text {ref }}\right) & =0 .
\end{aligned}
$$

The source current $\mathbf{j}^{s}$ is modeled by a mathematical dipole at position $\mathbf{x}_{0} \in \Omega$ with the moment $\mathbf{M} \in \mathbf{R}^{3}$

$$
\mathbf{j}^{s}(\mathbf{x})=\delta\left(\mathbf{x}-\mathbf{x}_{0}\right) \mathbf{M}
$$

The source has a singularity at $\mathbf{x}_{0}$ and is therefore difficult to model with standard finite elements. A modified subtraction method [16], [18], [19] is used to circumvent this problem, where the total potential is split into two parts

$$
\Phi=\chi \Phi^{\infty}+\Phi^{\mathrm{mod}}=F^{\infty}+\Phi^{\mathrm{mod}} .
$$

For convenience, we have defined the function

$$
F^{\infty}=\chi \Phi^{\infty} .
$$

The first part, $\Phi^{\infty}$, is the solution to (1) in an unbounded domain with constant conductivity $\sigma^{\infty}$

$$
\Delta \Phi^{\infty}=\frac{\nabla \cdot \mathbf{j}^{s}}{\sigma^{\infty}} .
$$

The solution can in this case be formed analytically [20] as

$$
\Phi^{\infty}(\mathbf{x})=\frac{1}{4 \pi \sigma^{\infty}} \frac{\left(\mathbf{x}-\mathbf{x}_{0}\right) \cdot \mathbf{M}}{\left|\mathbf{x}-\mathbf{x}_{0}\right|^{3}} .
$$


For future references, we notice that both the source current $\mathrm{j}^{s}$ and $\Phi^{\infty}$ depend linearly on the dipole moment $\mathbf{M} . \chi$ is a smooth cutoff function which is identically 1 in a neighborhood of $\mathbf{x}_{0}$. Using (4) and(1), the new formulation reads

$$
-\nabla \cdot\left(\sigma \nabla \Phi^{\mathrm{mod}}\right)=\nabla \cdot\left(\sigma \nabla F^{\infty}\right)-\nabla \cdot \mathbf{j}^{s} \text { in } \Omega
$$

subject to the conditions

$$
\begin{aligned}
\hat{\mathbf{n}} \cdot\left(\sigma \nabla \Phi^{\mathrm{mod}}\right) & =-\hat{\mathbf{n}} \cdot\left(\sigma \nabla F^{\infty}\right) \text { on } \partial \Omega \\
\Phi\left(\mathbf{x}_{\mathrm{ref}}\right) & =0 .
\end{aligned}
$$

Application of the standard FE method to (8) and (9) yield a linear equation system

$$
\mathbf{K} \mathbf{u}^{\bmod }=\mathbf{b}
$$

where $\mathbf{K} \in R^{N \times N}$ is a sparse symmetric positive definite stiffness matrix, $\mathbf{u}^{\text {mod }} \in R^{N}$ the coefficient vector of the modified electric potential and $\mathbf{b} \in R^{N}$ the right hand side vector with $N$ being the number of FE nodes.

In EEG applications the potentials are typically measured at approximately $40-100$ electrodes. Then the values of the potential at the electrodes can be obtained by multiplying the vector of nodes values with a restriction matrix $\mathbf{R}$

$$
\mathbf{u}_{\text {elec }}^{\text {mod }}=\mathbf{R} \mathbf{u}^{\text {mod }} .
$$

The size of the matrix $\mathbf{R}$ is $N_{\text {elec }} \times N$. Since only the relative differences of the potential are of interest, it is common in EEG to use the average signal as a common reference, this is the so-called average reference montage. Let $\overline{\mathbf{R}}$ be the transfer matrix such that

$$
\mathbf{u}_{\text {elec }}^{\text {mod }}-\overline{\mathbf{u}}_{\text {elec }}^{\text {mod }}=\overline{\mathbf{R}} \mathbf{u}^{\text {mod }}
$$

where $\overline{\mathbf{u}}_{\text {elec }}$ is the average of the potential at all nodes. $\overline{\mathbf{R}}$ can be obtained from $\mathbf{R}$ by subtracting the column-wise mean from each entry. From the relation (10) we see that $\mathbf{u}^{\text {mod }}=\mathbf{K}^{-1} \mathbf{b}$ and therefore

$$
\mathbf{u}_{\text {elec }}^{\text {mod }}-\overline{\mathbf{u}}_{\text {elec }}^{\text {mod }}=\overline{\mathbf{R}} \mathbf{u}^{\text {mod }}=\overline{\mathbf{R}} \mathbf{K}^{-1} \mathbf{b}=\overline{\mathbf{T}} \mathbf{b} .
$$

We call $\overline{\mathbf{T}}=\overline{\mathbf{R}} \mathbf{K}^{-1}$ the transfer matrix for the average reference montage. The right hand side, $\mathbf{b}$, is linear in the dipole moment $\mathbf{M}$ and nonlinear in dipole position $\mathbf{x}_{0}$, therefore we can write it as follows:

$$
\mathbf{b}=\mathbf{b}\left(\mathbf{x}_{0}, \mathbf{M}\right)=\mathbf{B}\left(\mathbf{x}_{0}\right) \mathbf{M} .
$$

In the modified subtraction method the contribution to the total potential comes from two parts. One part from the finite element method computed as described above and one direct contribution from (5). Both these contributions are linear in the dipole moment so therefore the total potential at the electrodes can be written

$$
\mathbf{u}_{\text {elec }}=\left(\overline{\mathbf{T}} \mathbf{B}\left(\mathbf{x}_{0}\right)+\mathbf{F}_{\text {elec }}^{\infty}\left(\mathbf{x}_{0}\right)\right) \mathbf{M}=\mathbf{G}\left(\mathbf{x}_{0}\right) \mathbf{M} .
$$

Here, $\mathbf{F}_{\text {elec }}^{\infty}\left(\mathbf{x}_{0}\right)$ is the value of the function $F^{\infty}$ for the three polarizations at all electrodes when the dipole is located at $\mathbf{x}_{0}$ and $\mathbf{G}\left(\mathbf{x}_{0}\right)$ is called the gain matrix. We use (15) in the inverse problem to find the position of the dipole.

\section{B. Inverse Problem}

Localization of the neural activity inside the brain based on the scalp EEG signal is called the EEG inverse problem. The problem is underdetermined (number of possible source locations $\gg$ number of electrodes) and the solution is nonunique. The remedy is to impose some regularization method on the source model as well as anatomical and physiological constraints as a priori knowledge about the source.

In a parametric method, the number of dipoles is assumed to be fixed and their locations and moments are chosen such that the potentials at the electrodes, $\mathbf{u}_{\text {elec }}$, that are computed according to (15), approximate the measured potentials $\mathbf{u}_{\text {meas }}$ well according to some criteria. Here we follow the common practice and choose the parameters such that we have the best fit in least squares sense. For one dipole we get the following minimization problem:

$$
J=\min _{\substack{\mathbf{x} \in \Omega_{\text {brain }} \\ \mathbf{M} \in \mathbf{R}^{d}}}\left\|\mathbf{u}_{\text {meas }}-\mathbf{G}(\mathbf{x}) \mathbf{M}\right\|
$$

where $\Omega_{\text {brain }}$ is the brain domain and $d$ the dimension. Since this is a least squares problem and $\mathbf{u}_{\text {elec }}$ depends linearly on the dipole moment it is convenient to separate the parameters in (16) and solve for the dipole moment $\mathbf{M}$ first. Define, for fixed $\mathbf{x} \in \Omega_{\text {brain }}$

$$
J(\mathbf{x})=\min _{\mathbf{M} \in \mathbf{R}^{d}}\left\|\mathbf{u}_{\text {meas }}-\mathbf{G}(\mathbf{x}) \mathbf{M}\right\| .
$$

According to the normal equations for linear least squares problems, optimality is obtained for

$$
\hat{\mathbf{M}}(\mathbf{x})=\left(\mathbf{G}^{T}(\mathbf{x}) \mathbf{G}(\mathbf{x})\right)^{-1} \mathbf{G}^{T}(\mathbf{x}) \mathbf{u}_{\text {meas }} .
$$

Substituting (18) into(17) yields after some manipulation

$$
J(\mathbf{x})=\left(\mathbf{u}_{\text {meas }}^{T}\left[\mathbf{I}-\mathbf{G}(\mathbf{x})\left(\mathbf{G}^{T}(\mathbf{x}) \mathbf{G}(\mathbf{x})\right)^{-1} \mathbf{G}^{T}(\mathbf{x})\right] \mathbf{u}_{\text {meas }}\right)^{1 / 2} .
$$

Now we can reduce (16) to a minimization problem only over the dipole position

$$
J=\min _{\substack{c_{\text {brain }}(\mathbf{x}) \leq 0, \mathbf{x} \in \Omega_{\text {brain }}}} J(x)
$$

where a constraint function $c_{\text {brain }}$ is introduced to define the optimization domain [16]. Here, the optimization problem is a function of the source position only, thus the complexity of the inverse problem is reduced.

Moreover, this approach can be used to estimate more than one source. To choose the number of dipoles, one can start with one dipole and then increase the number until some criterion on the matching between the measured and optimized potentials at the electrodes is met. One natural criterion is that the relative difference $\left\|\mathbf{u}_{\text {meas }}-\mathbf{u}_{\text {opt }}\right\| /\left\|\mathbf{u}_{\text {meas }}\right\|$ is lower than some prescribed value.

\section{Particle Swarm Optimization}

\section{A. Standard Particle Swarm Optimization}

The Particle Swarm Optimization concept was first introduced by Kennedy and Eberhart [21], [22] in 1995 based on the social system behavior such as the movement of flock of birds 
or a school of fish when searching for food. Each individual in the swarm is called a particle. The $i$ th particle of the swarm is represented by the vectors $\mathbf{X}_{i}$ for its position and $\mathbf{V}_{i}$ for its velocity. The particle has a memory to record the position of its previous best performance, personal best (pbest), in the vector $\mathbf{P}_{i}$ and the position of the best particle in the swarm, global best (gbest), which is recorded in the vector $\mathbf{P}_{g}$. The particle swarm optimization algorithm consists of, in each iteration, changing the velocity of each particle towards the position of its best performance, $\mathbf{P}_{i}$, and the swarm best position, $\mathbf{P}_{g}$. Thus in the original version particles move according to the following formula:

$$
\left\{\begin{array}{cc}
\mathbf{V}_{i}^{t+1}= & \mathbf{V}_{i}^{t}+c_{1} \operatorname{Rand}()\left(\mathbf{P}_{i}-\mathbf{X}_{i}^{t}\right) \\
& +c_{2} \operatorname{Rand}()\left(\mathbf{P}_{g}-\mathbf{X}_{i}^{t}\right) \\
\mathbf{X}_{i}^{t+1}= & \mathbf{X}_{i}^{t}+\mathbf{V}_{i}^{t+1} .
\end{array}\right.
$$

Parameters $c_{1}$ and $c_{2}$ are the cognitive and social learning rates . These two rates control the relative influence of the memory of the swarm best performance to the memory of the individual and are often selected to the same value to give each learning rate equal weight. In addition to the $c_{1}$ and $c_{2}$ parameters, implementation of the original algorithm also requires placing limits on the search area $\left(\mathbf{X}_{\max }\right.$ and $\left.\mathbf{X}_{\min }\right)$, and the velocity $\left(\mathbf{V}_{\max }\right)$.

Shi and Eberhart [23], [24] devised an inertia weight, $w$, to improve the accuracy of the PSO by damping the velocities over time, allowing the swarm to converge with greater precision. By integration of $w$ into the algorithm, the formula for computing the new velocity is

$$
\begin{aligned}
\mathbf{V}_{i}^{t+1}=w \mathbf{V}_{i}^{t}+c_{1} \operatorname{Rand}()\left(\mathbf{P}_{i}-\right. & \left.\mathbf{X}_{i}^{t}\right) \\
& +c_{2} \operatorname{Rand}()\left(\mathbf{P}_{g}-\mathbf{X}_{i}^{t}\right) .
\end{aligned}
$$

As originally developed, $w$ is often decreased linearly from about 0.9 to 0.4 during a run. Suitable selection of the inertia weight provides a balance between exploration, the ability to test various regions in the problem space in order to locate a good optimum, hopefully the global one, and exploitation, the ability to concentrate the search around a promising candidate solution in order to locate the optimum precisely [24].

The PSO algorithm introduced by Kennedy and Eberhart has been proven to be powerful but needs to select various parameters, such as the maximum velocity coefficient, the swarm size, the neighborhood size as well as the cognitive and social learning rates. A complete theoretical analysis of the algorithm has been given by Clerc and Kennedy [25]. Based on this analysis, the authors derived a reasonable set of tuning parameters, as confirmed by [26]. However, the parameter selection in a specific problem is not straightforward. The PSO algorithm has a risk to trap in a local minima and lose its exploration-exploitation ability [27]. In the following section, we describe a modified PSO (MPSO) algorithm [16], which can help to cure the aforementioned drawbacks.

\section{B. Modified Particle Swarm Optimization}

One way to avoid PSO to trap in local minima is mutation and using evolutionary programming (EP). In this method $M$ particles are selected among the swarm population by the $q$-tournament selection method and then mutated by the EP method [28].
By evaluating the fitness value of all the particles, the global best position is determined. For each particle, the nearest elite particle is determined by the Euclidean distance. The velocity and the position of the particles are updated according to the global best position, the nearest elite position, and the personal best position. These are applied to the PSO with inertia weight as follows:

$$
\begin{aligned}
\mathbf{V}_{i}^{t+1}=w \mathbf{V}_{i}^{t} & +c_{1} \operatorname{Rand}()\left(\mathbf{P}_{i}-\mathbf{X}_{i}^{t}\right) \\
& +c_{2} \operatorname{Rand}()\left(\mathbf{P}_{g}-\mathbf{X}_{i}^{t}\right) \\
& +c_{3} \operatorname{Rand}()\left(\mathbf{P}_{e}-\mathbf{X}_{i}^{t}\right)
\end{aligned}
$$

where $c_{3}$ denotes the constant of the nearest elite and $\mathbf{P}_{e}$ the nearest elite position.

In the MPSO, we introduced the concept of authority to maintain the exploration ability and increase the exploitation ability. In the concept of authority, as the swarm moves close to a minima the $R$ closest particles to gbest are extracted and they are allowed to fly freely based on their memory and knowledge. Thus, the velocity update is divided into two parts as

$$
\begin{array}{r}
\mathbf{V}_{i}^{t+1}=w \mathbf{V}_{i}^{t}+c_{1} \operatorname{Rand}()\left(\mathbf{P}_{i}-\mathbf{X}_{i}^{t}\right)+c_{2} \operatorname{Rand}()\left(\mathbf{P}_{g}-\mathbf{X}_{i}^{t}\right) \\
+c_{3} \operatorname{Rand}()\left(\mathbf{P}_{e}-\mathbf{X}_{i}^{t}\right)
\end{array}
$$

where $i=1,2, \ldots, N-R$ and

$$
\mathbf{V}_{r}^{t+1}=w \mathbf{V}_{r}^{t}+c_{1} \operatorname{Rand}()\left(\mathbf{P}_{r}-\mathbf{X}_{r}^{t}\right)
$$

where $\mathbf{V}_{r}, \mathbf{X}_{r}$, and $\mathbf{P}_{r}$ are the velocity, position and personal best of the $r$ th particle, respectively, for $r=N-R+1, \ldots, N$. The $R$ nearest particles to gbest are reselected in each iteration to ensure that the particles which moved away from the gbest lose their authority and next iteration update their velocity based on (24). It means that in some steps the particles which are closer to the global best can influence the performance and decision of the swarm more than others. The concept of authority allows the swarm to have more information around gbest before lots of particles come close to it and stuck with each other, thus it improves the exploitation ability. The concept of authority mixed with EP helps to keep the balance of the exploration-exploitation trade-off as well as to avoid trapping in local minima.

A natural choice for $R$ is the number of neighborhoods for each particle in the swarm, which in our case is equal to 5 . The concept of authority allows the swarm to have more information around gbest before lots of particles approach it and get stuck to each other, thus it improves the exploitation ability. The concept of authority mixed with EP helps to keep the balance between exploration and exploitation as well as avoiding trapping in local minima.

\section{The MPSO Parameter Selection}

Usually, the swarm size is constant. Some authors use 20, while some others use 30 [22], [24], but nobody has proved that one given size is really better than any other. Thus it seems better to let the algorithm modify the swarm size [29], adaptively based on the current situation. In each iteration, the swarm has information about each particle's position, $\mathbf{X}_{i}$, personal best, $\mathbf{P}_{i}$, velocity, $\mathbf{V}_{i}$ as well as the previous objective function values and improvement of the objective function, 
$\Delta J_{i}$ since last check (difference between the previous objective function value and the current one). The swarm also has some global information, i.e., the size and time step. Using this information, the swarm has two options to act on particles. It may remove particles from the swarm or generate new particles. The condition for the swarm to change the status of a particle is based on the following situations.

- If one particle has had enough improvement a new particle is generated from that particle and the old one is kept.

- If one particle has not had enough improvement that particle is removed from the swarm.

Here, the status changes every five iterations which is equal to the neighborhood size. The enough improvement is defined by "improvement for 5 iterations". Reflecting walls are used as boundary conditions for MPSO. When a particle hits the boundary in one of the dimensions, the sign of that velocity component is changed and the particle is reflected back towards the solution space. This boundary condition keeps the particles inside the searching space. Here, we use the following parameters coefficients: $w=$ linear from 0.9 to $0.4, c_{1}=0.8, c_{2}=0.4$, $c_{3}=0.8$, and initial swarm size $=30$, which are suggested in the literature [24], [28].

\section{Bound and Anatomical Constraints}

Because of the dipole singularity, the FEM modeling usually has high error for sources located close to interfaces between tissues of different conductivities [30]. This causes false extrema that can trap the optimization methods in local minima during the minimization process. To avoid such problems at the interfaces we will use a constraint for the MPSO search method. The position of each particle is checked before the evaluation. If the particle either is placed on a interface or its distance to an interface is less than half a mesh size, then the particle is replaced to the middle of the mesh cell it is located in. With this position correction we can be sure that the particles are a safe distance away from the interfaces.

Restricting the search space in limited areas of the brain volume (e.g., the gray matter) reduces the ambiguity of source localization [31]. In the inverse problem we consider only those dipole locations and orientations that are consistent with the anatomical data. The EEG signals are generated by currents flowing in the apical dendrites of cortical pyramidal cells [32], [33] so the searching area could be restricted only to the cortex sheet of the brain. We use this information and add it to MPSO searching. For the anatomical constraint MPSO solely evaluates(19) for the particles which are placed in the gray matter and assign a high value to others. The MPSO starts from gray matter and in this way it ends up in the gray matter, also this constraint helps to avoid trapping the inverse problem in false local minima in other tissues. This reduces the number of evaluations and thus the computational time significantly.

\section{MRI AND EEG DATA}

\section{A. Head Model and Sensor Positioning}

Structural MR images were acquired using a PHILIPS ACHIEVA 3T scanner (Sahlgrenska University Hospital,

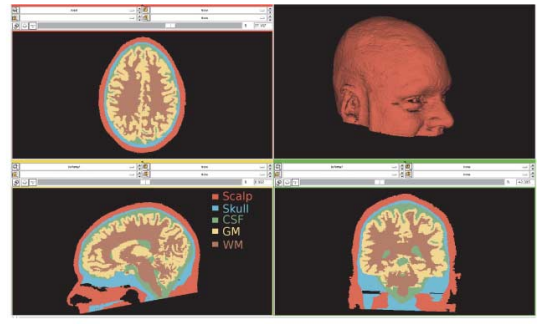

(a)

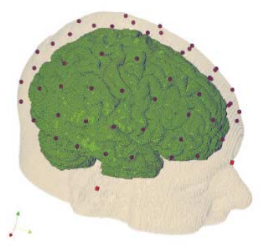

(b)

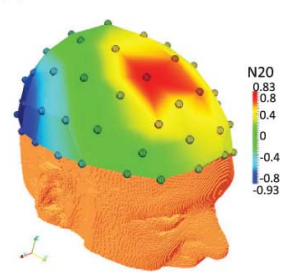

(c)
Fig. 2. (a) The manually segmented MRI for five tissues, (b) the subject's FEM gray matter and skin generated from the segmented MRI and the registered EEG electrode positions on the scalp surface, and (c) 61 EEG electrodes topography for the N20 peak.

Gothenburg, Sweden) equipped with a 32 channel head coil. T1-weighted images were acquired for a healthy subject, (192 sagittal slices, matrix size $=256 \times 256$, voxel size $=0.9375 \mathrm{~mm}^{3}$, flip angle $8^{\circ}, \mathrm{TR} / \mathrm{TE}=2.300 / 2.98 \mathrm{~ms}$ ).

Since the authors' previous study [34] showed that the widely used softwares packages for brain segmentation, namely FSL [35] and FreeSurfer [36] cause significant errors in source localization results, here the segmentation of the five tissues, i.e., gray matter, white matter, cerebrospinal fluid (CSF), skull, and skin was done manually by a clinical expert. Our expert spent $170 \mathrm{~h}$ to fully segment the head model (120 slices).

The mesh model which is generated from the cubic voxel structure of the MR image has good numerical properties [37] and it simplifies FE mesh generation as well. Here, the high resolution model is necessary to accurately model the CSF compartment and the thin area of the skull. We generated the $1 \mathrm{~mm}$ hexahedra FE head model with approximately 2.8 million nodes from the segmented MRI [see Fig. 2(b)]. The following conductivities were then assigned to the FE compartments based on their segmentation labels and the isotropic reference model [38], [39]: skin $=0.43 \mathrm{~S} / \mathrm{m}$, skull $=0.0042 \mathrm{~S} / \mathrm{m}$ (skull to skin conductivity ratio of approximately $1: 100), \mathrm{CSF}=1.538 \mathrm{~S} / \mathrm{m}$, gray matter $=0.33 \mathrm{~S} / \mathrm{m}$, and white matter $=0.142 \mathrm{~S} / \mathrm{m}$. Sixty-one EEG electrodes were placed on the subject head based on the 10/10 EEG electrode system [40]. The 3-D (-x,-y,-z) coordinates of these electrodes were measured before and after the SEP stimulation experiment with a digitizer and for the electrode registration three reference points, i.e., nasion (the delve at the top of the nose, level with the eyes), left tragus (small point situated in front of the left concha), and right tragus (small point situated in front of the right concha) were measured on the subject head as well. Then by using an affine transformation, the measured points were co-registered to the surface of the FE model. 


\section{B. Somatosensory Evoked Potential}

Evoked potentials are the electrical signals generated by the nervous system in response to sensory stimuli. Auditory, visual, and somatosensory stimuli are commonly used for clinical evoked potential studies. SEP consist of a series of waves that reflect sequential activation of neural structures along the somatosensory pathways. Sensory nerves (cell bodies in the dorsal root ganglia) transmit the signal rostrally and ipsilaterally (firstorder fibers), in the posterior column to a synapse in the dorsal column nuclei at the cervicomedullary junction [41]. Then the signal is passed via the second order fibers that cross to the contralateral thalamus via the medial lemniscus. Finally, the signal travels via the third-order fibers from the thalamus to the frontoparietal sensory cortex. While SEP can be elicited by mechanical stimulation, clinical studies use electrical stimulation of peripheral nerves, which gives larger and more robust responses. The stimulation sites typically used for clinical diagnostic SEP studies are the median nerve at the wrist, the common peroneal nerve at the knee, and/or the posterior tibial nerve at the ankle.

In this study two sets of stimulations were measured. 1) Median nerve at the left wrist. The anode was placed just proximal to the palmar crease, and the cathode was placed between the tendons of the palmaris longus muscle, $3 \mathrm{~cm}$ proximal to the anode. The selected nerves were stimulated with monophasic square pulses, $300 \mu \mathrm{s}$ in duration and the stimuli were delivered by using a constant current stimulator with $4.8 \mathrm{~mA}$. 2) The left posterior tibial nerves at the subject's ankle. The selected nerves were stimulated with monophasic square pulses, $300 \mu$ s in duration and the stimuli was delivered by using a constant current with $5.2 \mathrm{~mA}$. The SEP components typically are named by their polarity and typical peak latency in the normal population. For example, N20 is a negativity that typically peaks at $20 \mathrm{~ms}$ after the stimulus.

\section{EEG Signal Preprocessing}

The EEGs of a healthy subject, used for the EEG source localization, were recorded at the Department of Clinical Neurosciences of the Sahlgrenska University Hospital (Gothenburg, Sweden). The participant (30 years old) was without substance abuse or dependence and had no known neurological or psychiatric illness or trauma. A 61-channel EEG system was used at a sampling frequency of $2 \mathrm{KHz}$. The EEG time series were filtered (FIR, band-pass of $1-45 \mathrm{~Hz}$ and notch of $50 \mathrm{~Hz}$ ), rereferenced against the common average reference, and segmented into nonoverlapping $300 \mathrm{~ms}$ epochs using the EEGLab software [42]. Artifacts in all channels were edited offline: first automatically, based on an absolute voltage threshold $(100 \mathrm{mV})$ and on a transition threshold $(50 \mathrm{mV})$, and then on the basis of a thorough visual inspection. The sensors with high artifacts were removed from the recorded signals. Using short segments for analysis allowed us to record 160 artifact-free epochs per subject in order to achieve high confidence of the data. The epochs were selected based on maximization of the SNR and to increase the SNR signal averaging of 160 artifact-free epochs were used. After averaging the SNR was equal to $28 \mathrm{~dB}$. Then the peak of the averaged signals was used as input for the inverse problem.

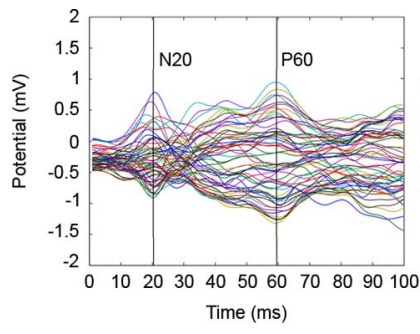

(a)

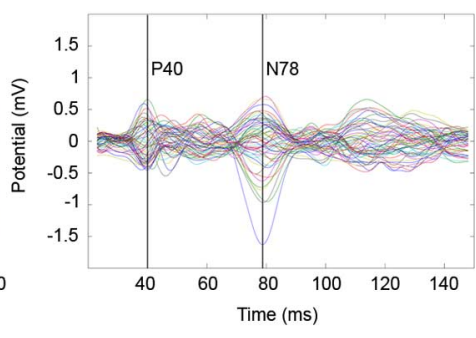

(b)
Fig. 3. SEP: Butterfly plot of the averaged (a) median nerve stimulation N20 and its late cortical activity P60 and (b) tibial nerve stimulation N40 and its late cortical activity N78 at the 61 EEG electrode positions.

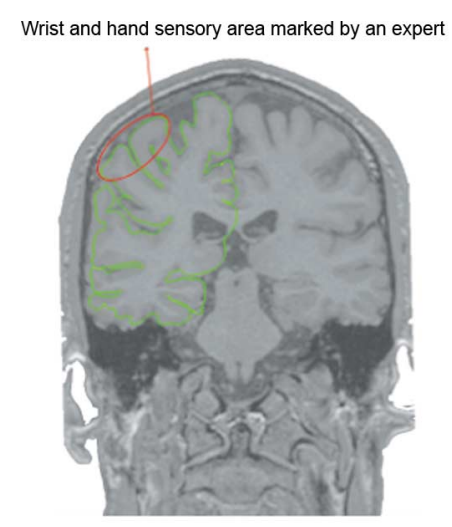

(a)

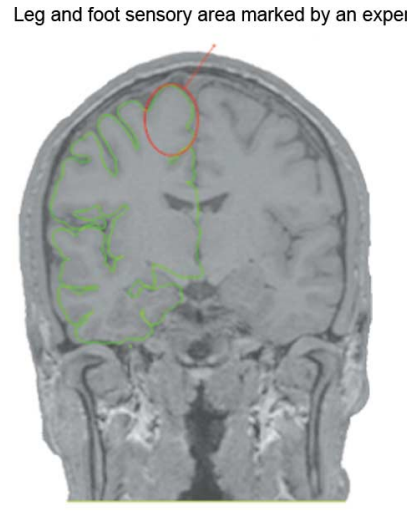

(b)
Fig. 4. Somatosensory cortex: the location and size of the subject's somatosensory cortical area corresponding to the left wrist (a) and leg (b) stimulations are marked by a clinical expert.

\section{RESUltS AND DiscusSION}

\section{A. Validation}

Validation of the source localization is difficult, because there exist no "ground truth" to compare with and also we do not have access to other functional modalities, e.g., fMRI and MEG data from our subject for the same kind of stimulation. We have taken two approaches to validate our method: first we use the physiological knowledge on localization of motor and sensory functions [43] based on clinical expertise and second we do an exhaustive search pattern, i.e., inversion was performed for each possible source location in the motor and sensory cortex area inside a region of interest (ROI), and the location producing the smallest residual norm was selected as the best possible source location. In [44] it was shown that median nerve SEPs (N20) are generated by primary somatosensory cortex in the posterior wall of the central fissure (SI area) and later cortical waves recorded at P60 are more susceptible to changes by cognitive factors. Moreover, several studies [45], [46] have shown that the strong recorded signals in the primary somatosensory cortex correspond to N20 and P40 for median and tibial nerve, respectively. Fig. 3 shows the average of 160 stimulations for median (a) and tibial (b) nerve stimulation at the 61 EEG electrodes, respectively. Fig. 4 shows a visual image of locations and sizes of the subject's somatosensory cortical areas for the left wrist and leg. 
For the single point source the cost function (relative error) is defined as

$$
\text { Relative Error }=\frac{\left\|\mathbf{u}_{\text {meas }}-\mathbf{u}_{\text {est }}\right\|}{\left\|\mathbf{u}_{\text {meas }}\right\|}
$$

where $\mathbf{u}_{\text {meas }}$ is a vector of the measured potentials on the scalp, $\mathbf{u}_{\text {est }}$ is a vector of the potential generated by the estimated dipole and $\|\cdot\|$ denotes the Euclidean norm. For the exhaustive search we selected a large area that covered all of the left and right hemisphere motor and somatosensory cortex. This ROI has approximately 110000 voxels. All computations were performed on an Intel $2.93 \mathrm{GHz}$ workstation with 8GB RAM memory and the post processing and visualizations were done using Matlab (R2012a) and the 3DSlicer (3.6.3) software [47].

\section{B. The MPSO Results}

In [16] we showed that the proposed MPSO method converges faster and is less prone to be trapped in local minima compared to some other PSO methods in the literature. Therefore, we only use MPSO in this section. We ran the source localization method for $\mathrm{N} 20$ and $\mathrm{P} 40$ signals recorded from median and tibial nerves as well as their later cortical waves i.e., P60 and N78 [44], respectively (see Fig. 3). For the MPSO, 30 particles were initialized in the ROI, which cover the left and right hemisphere motor and somatosensor cortex. Each particle had six parameters, three parameters for the position and three parameters for the orientation. As the proposed MPSO has the ability to use an adaptive swarm size the number of particles initialized is not crucial for the convergence. These particles were generated randomly inside the gray matter with uniform distribution. Moreover, the boundary and anatomical constraints (see Section III-D) were applied to the MPSO. The optimization method was terminated when the minimum relative error from the exhaustive search was obtained. Fig. 5 shows the convergence curve for the MPSO for all cases. Table I shows the number of evaluations and the relative error between measured and estimated potentials for each case.

Figs. 6 and 7 show the measured and estimated scalp surface potential topographies for the median nerve stimulation and its late cortical activity, respectively. Figs. 8 and 9 show the estimated source position for median nerve stimulation and its late cortical activity on the subject's segmented MR image.

Figs. 10 and 11 show the measured and estimated scalp surface potential topographies for the tibial nerve stimulation and its late activity, respectively. Figs. 12 and 13 show the estimated source position for the tibial nerve stimulation and its late cortical activity on the subject's segmented MR image.

We have run both median nerve stimulation (N20) and tibial nerve stimulation (P40) for two dipole sources. We kept the first estimated dipole fixed and then started searching for the second dipole which clearly has weaker amplitude. For N20 the relative error decreased from 0.23 to 0.18 and for P40 it decreased from 0.23 to 0.19 .

\section{CONCLUSION}

In this paper, the ability to perform EEG source localization was tested with a new optimization method. The new method is

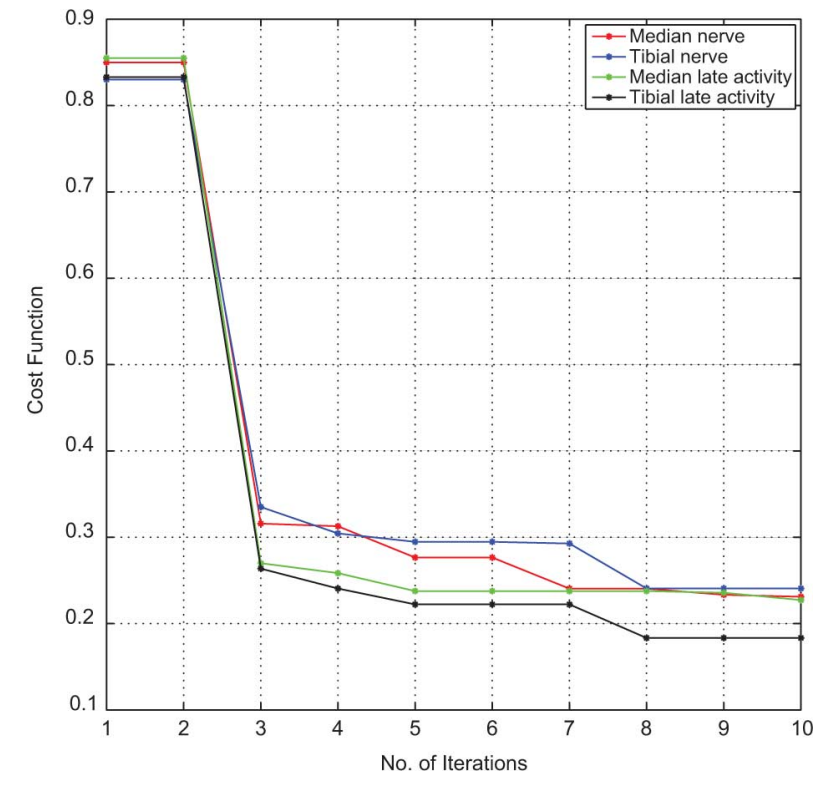

Fig. 5. MPSO convergence curve.

TABLE I

NuMBER OF VALUATIONS AND RELATIVE ERROR FOR BOTH THE MPSO AND Exhaustive Search Methods. MPSO and Exhaustive SEarch GaVe IDENTICAL RESUlT BUT WITH A MUCH LOWER NuMBER OF EVALUATION FOR MPSO

\begin{tabular}{|c|c|c|c|c|}
\hline & N20 & P60 & P40 & N78 \\
\hline No. of eval. for MPSO & 300 & 300 & 310 & 320 \\
\hline No. of eval. for Exh. search & 110000 & 110000 & 110000 & 110000 \\
\hline Relative error & 0.2311 & 0.2407 & 0.2273 & 0.1834 \\
\hline
\end{tabular}

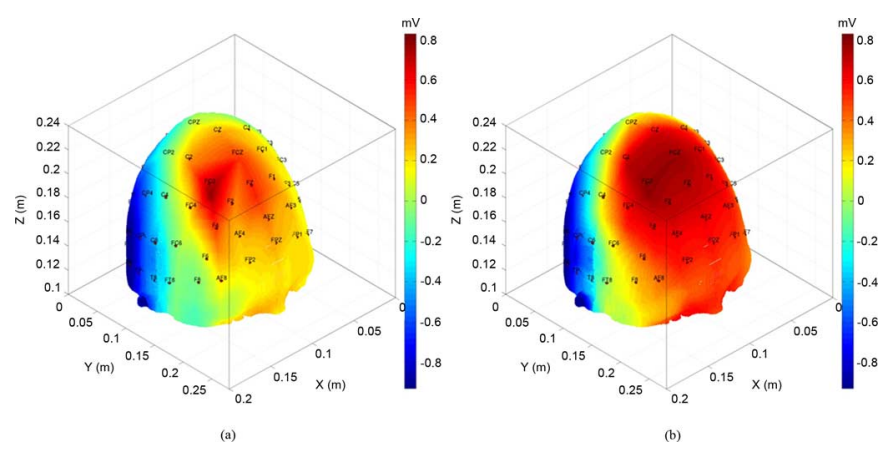

Fig. 6. Scalp surface potential topography for the median nerve stimulation (N20). (a) Measured EEG and (b) estimated EEG potential.

a modified version of particle swarm optimization. In this new approach, positions and orientations of dipoles are optimized to obtain the best least squares fit between the measured EEG signals and simulations. For the forward problem, we built a realistic high-resolution finite element head volume conductor based on a T1-weighted MR dataset including five tissues, i.e., gray matter, white matter, CSF, skull, and skin.

SEPs stimulation by an electrical pulse on the median nerve of a healthy subject, was recorded with 61 EEG electrodes placed on the scalp. Based on physiological knowledge, the somatosensory cortex generates the SEP signals and its position is known a priori to a good approximation. Although this position slightly differs between individuals the variations are small [43] and we can use this knowledge to validate 


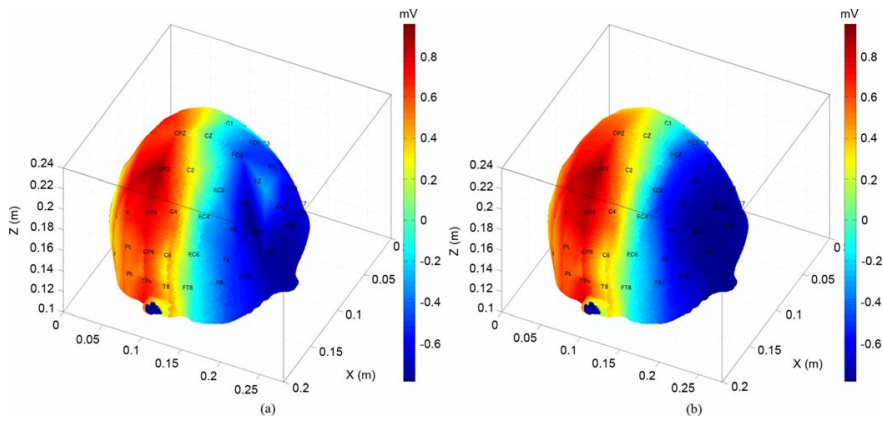

Fig. 7. Scalp surface potential topography for the late cortical activity of the median nerve stimulation (P60). (a) Measured EEG and (b) estimated EEG.

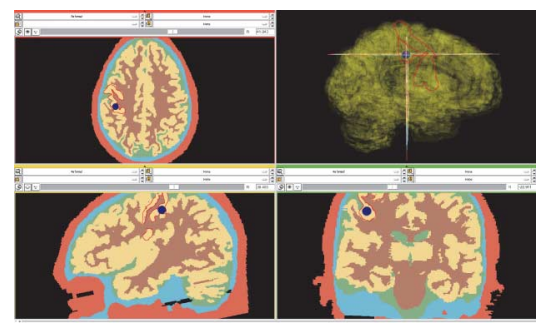

Fig. 8. Estimated source position for median nerve stimulation (N20) placed on the subject's segmented MR image. The 3-D view shows the estimated source placed inside the gray matter. Red lines are central sulcus (SI).

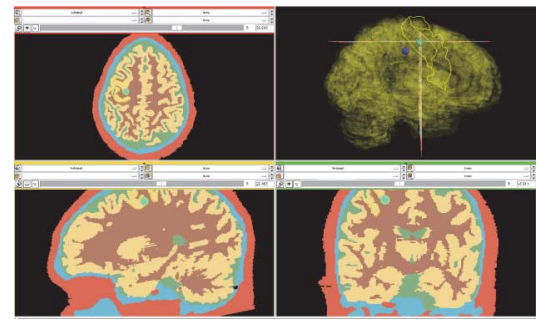

Fig. 9. The estimated source position for late cortical activity of median nerve stimulation (P60) placed on the subject's segmented MR image. The 3-D view shows both estimated sources of median nerve (N20, dark blue), and late cortical activity (P60, cyan), placed inside the gray matter. Yellow lines are primary motor cortex (MI).
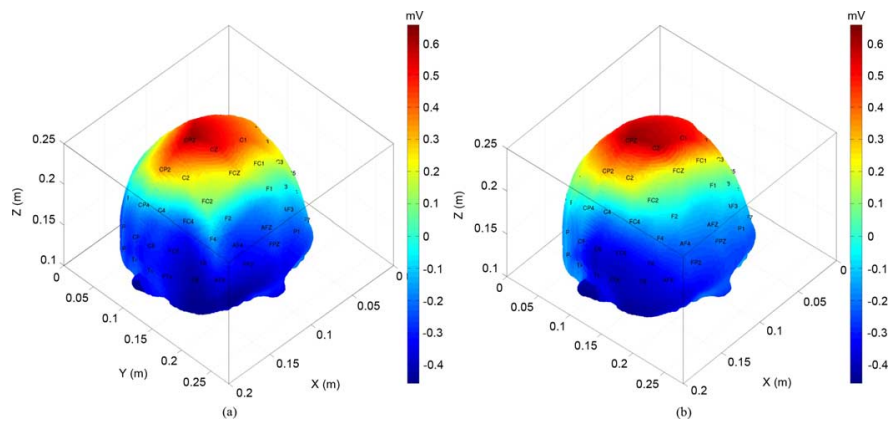

Fig. 10. Scalp surface potential topography for the tibial nerve stimulation (N40). (a) measured EEG and (b) estimated EEG potential.

our results. Moreover, an exhaustive search pattern was performed for each possible source location in the motor and sensory cortex and used for validation of the MPSO results. We have applied MPSO to median (N20) and tibial (P40) nerve stimulations as well as their late cortical activities, (P60) and

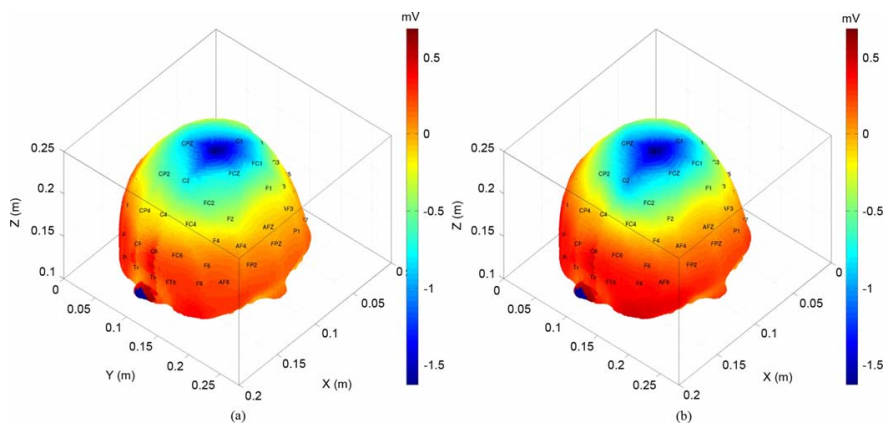

Fig. 11. Scalp surface potential topography for the late cortical activity from tibial nerve stimulation (N78). (a) measured EEG and (b) estimated EEG potential.

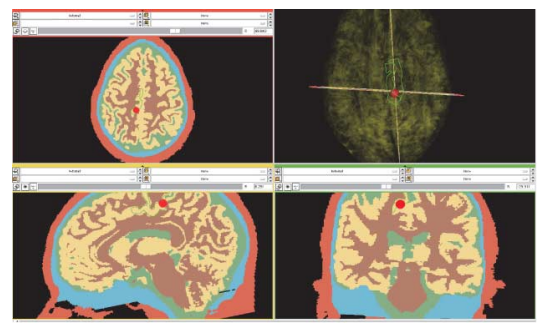

Fig. 12. The estimated source position for tibial stimulation from P40 placed on the subject's segmented MRI. The 3-D view shows the estimated source placed inside the gray matter. Green lines are sensory and motor cortex for the leg area.

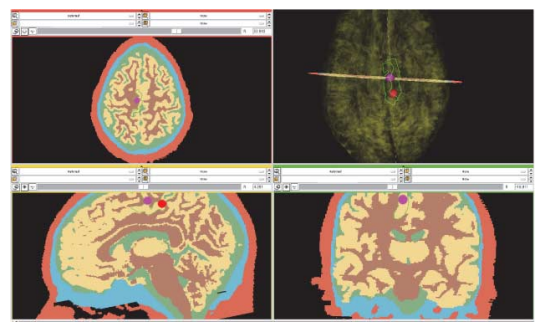

Fig. 13. The estimated source position for late cortical activity of tibial nerve stimulation (N78) placed on the subject's segmented MR image. The 3-D view shows both estimated sources of tibial nerve (P40), dark red, and late cortical activity (N78), violet, placed inside the gray matter. Green lines are sensory and motor cortex for the leg area.

(N78), respectively. Comparison between the recorded EEG and estimated scalp potential topographies showed a good agreement in all cases. Moreover, based on clinical expertise the estimated sources are located in correct region. The EEG source localization results obtained from MPSO gave the same results as an exhaustive search but with significantly lower computational complexity.

Electrical stimulation of the tibial nerve generated a source at primary sensory (SI) and the source moved to the primary motory (MI) for the evoked late cortical activity EEG signal. The source of activated cortex in MI was $13 \mathrm{~mm}(-y)$ anterior, $11 \mathrm{~mm}(-x)$ medial, and $6 \mathrm{~mm}(-z)$ superior of activated cortex in SI. This results agree with the nonpainful event-related fMRI study presented in [48] and the MEG study on the difference in responses to somatosensory electrical stimuli between primary and secondary (SII) sensory presented in [49].

Electrical stimulation of the median nerve evoked a significant EEG signal increase in contralateral SI. The late cortical 
activity on the other hand evoked a significant EEG signal increase in primary motor cortex (MI). The source of activated cortex in MI was $13 \mathrm{~mm}(-y)$ anterior, $10 \mathrm{~mm}(-x)$ medial, and $15 \mathrm{~mm}(-z)$ superior of activated cortex in SI. These results are similar to those found in a recent MEG [50] and fMRI study [45], which allocated the second peak of the magnetic field evoked by median nerve stimulation into MI.

The segmentation approaches proposed to date for EEG source localization [51], [52] all require manual intervention. Consequently the segmentation process is laborious, time-consuming, and subjective. Future work will include development of methods for automatic multi-tissue segmentation of MRI [53]. However, even though more work is needed to improve the preprocessing steps a fast and reliable method for the source localization is crucial and e.g., a necessity to enable the use of source localization in future EEG-guided rTMS applications [54].

\section{ACKNOWLEDGMENT}

The authors would like to thank Prof. M. Elam from the Sahlgrenska University Hospital for his help to measure the data and S. Bergstrand from the Sahlgrenska University Hospital for all his kind help during the result analysis as well as being subject for the measurement. The authors would like to thank to Assoc. Prof. J. Gellermann for her manual segmentation of the MR images.

\section{REFERENCES}

[1] U.S. Centers for Disease Control and Prevention, Coordinating Center for Health Promotion, U.S. Department of Health and Human Services, "Targeting epilepsy one of the nations most common disabling neurological condition," Jan. 2008.

[2] M. J. England, C. T. Liverman, A. M. Schultz, and L. M. Strawbridge, "Epilepsy Across the Spectrum: Promoting Health and Understanding," in Committee on the Public Health Dimensions of the Epilepsies; Board on Health Sciences Policy; Institute of Medicine, Ed. Atlanta, GA: Nat. Acad. Press, 2012.

[3] D. L. Kraeme, M. E. Brandling-Bennett, and D. G. Vossler, "Epilepsy surgery," Medscape Ref., Drug, Diseases Procedures, 2011.

[4] H. Laufs, "Functional imaging of seizures and epilepsy: Evolution from zones to networks," Current Opin. Neurol., vol. 25, 2012.

[5] B. Vanrumste, G. V. Hoey, R. V. de Walle, M. R. D'Have, I. A. Lemahieu, and P. A. Boon, "Comparison of performance of spherical and realistic head models in dipole localization from noisy EEG," Med. Eng. Phys., vol. 24, no. 6, pp. 403-418, 2002.

[6] T. Heinonen, H. Eskola, P. Dastidar, P. Laarne, and J. Malmivuo, "Segmentation of T1 MR scans for reconstruction of resistive head models," Comput. Methods Programs Biomed., vol. 54, no. 3, pp. 173-181, 1997.

[7] P. Basser, J. Mattiello, and D. LeBihan, "MR diffusion tensor spectroscopy and imaging," Biophys. J., vol. 66, no. 1, pp. 259-267, 1994.

[8] J. C. De Munck, "The potential distribution in a layered anisotropic spheroidal volume conductor," J. Appl. Phys., vol. 64, no. 2, pp. 464-470, 1988.

[9] C. C. Wood, "Application of dipole localization methods to source identification of human evoked potentials," Ann. NY Acad. Sci., vol. 388 , no. 1, pp. 139-155, 1982.

[10] D. W. Marquardt, "An algorithm for least-squares estimation of nonlinear parameters," J. Soc. Indust. Appl. Math., vol. 11, pp. 431-441, 1963.

[11] J. Nelder and R. Mead, "A simplex method for function minimization," Comput. J., vol. 4, pp. 308-313, 1965.

[12] D. Z. Yao, Electric theory and techniques of brain functional prospecting (in Chinese). Beijing, China: Beijing: Sci. Press, 2003.

[13] R. G. M. D. C. Fernandez and S. Gonzalez, "Some limitations of spatio temporal source models," Brain Topogr., vol. 7, no. 3, pp. 233-243, 1995.
[14] Z. J. Koles, "Trends in EEG source localization," Electroencephalogr. Clin. Neurophysiol., vol. 106, 1998.

[15] R. G. Menendez and S. L. Gonzalez, "Single dipole localization: Some numerical aspects and a practical rejection criterion for the fitted parameters," Brain Topogr., vol. 6, no. 4, pp. 277-282, 1994.

[16] Y. Shirvany, F. Edelvik, S. Jakobsson, A. Hedström, and M. Persson, "Application of particle swarm optimization in epileptic spike EEG source localization," Appl. Soft Comput., vol. 13, no. 5, pp. 2515-2525, 2013.

[17] Y. Shirvany, F. Edelvik, S. Jakobsson, A. Hedström, Q. Mahmood, A. Chodorowski, and M. Persson, "Non-invasive EEG source localization using particle swarm optimization: A clinical experiment," in Proc. Annu. Int. Conf. IEEE Eng. Med. Biol. Soc., 2012, pp. $6232-6235$

[18] S. van den Broeh, H. Zhou, and M. Peters, "Computation of neuromagnetic fields using finite-element method and Biot-Savart law," Med. Biol. Eng. Comput., vol. 34, no. 1, pp. 21-26, 1996.

[19] F. Edelvik, B. Andersson, S. Jakobsson, S. Larsson, M. Persson, and Y. Shirvany, "An improved method for dipole modeling in EEG-based source localization," World Congr. Med. Phys. Biomed. Eng., vol. 25, pp. 146-149, 2009.

[20] C. H. Wolters, M. Kuhn, A. Anwander, and S. Reitzinger, "A parallel algebraic multigrid solver for finite element method based source localization in the human brain," Comput. Visualizat. Sci., vol. 5, no. 3, 2002.

[21] R. Eberhart and J. Kennedy, "A new optimizer using particle swarm theory," in Proc. 6th Int. Symp. Micro Mach. Human Sci., 1995, pp. 39-43.

[22] J. Kennedy and R. Eberhart, "Particle swarm optimization," in Proc., IEEE Int. Conf. Neural Netw., 1995, vol. 4, pp. 1942-1948.

[23] Y. Shi and R. Eberhart, "A modified particle swarm optimizer," in Proc. IEEE Int. Conf. Evolut. Computat. Proc., IEEE World Congr. Computat. Intell., 1998, pp. 69-73.

[24] Y. Shi and R. C. Eberhart, "Parameter selection in particle swarm optimization," in Proc. 7th Int. Conf. Evolut. Program,, London, U.K., 1998, pp. 591-600.

[25] M. Clerc and J. Kennedy, "The particle swarm: Explosion, stability, and convergence in a multidimensional complex space," Trans. Evol. Comp., vol. 6, no. 1, pp. 58-73, 2002.

[26] R. C. Eberhart and Y. Shi, "Comparing inertia weights and constriction factors in particle swarm optimization," in Proc. 2000 Cong. Evolut. Comput., 2000, vol. 1, pp. 84-88.

[27] P. J. Angeline, "Using selection to improve particle swarm optimization," in Proc. Evolut. Comput., 1998, pp. 84-89.

[28] K. B. Lee and J. H. Kim, "Particle swarm optimization driven by evolving elite group," in IEEE Cong. Evolut. Comput., 2009, pp. 2114-2119.

[29] M. Clerc, Think locally, act locally: The way of life of cheap-PSO, an adaptive PSO Tech. Rep., 2001.

[30] K. Awada, D. Jackson, J. Williams, D. Wilton, S. Baumann, and A. Papanicolaou, "Computational aspects of finite element modeling in EEG source localization," IEEE Trans. Biomed. Eng., vol. 44, no. 8 , pp. 736-752, Aug. 1997.

[31] A. M. Dale and M. I. Sereno, "Improved localization of cortical activity by combining EEG and MEG with MRI cortical surface reconstruction: A linear approach," J. Cogn. Neurosci., vol. 5, pp. 162-176, 1993.

[32] S. Murakami and Y. Okada, "Contributions of principal neocortical neurons to magnetoencephalography and electroencephalography signals," J. Physiol., vol. 575, no. 3, pp. 925-936, 2006.

[33] L. Kiloh, A. McComas, J. Osselton, and A. Upton, Clin. Electroencephalogr., 1981.

[34] Y. Shirvany, A. Porras, K. Kowkabzadeh, Q. Mahmood, H.-S. Lui, and M. Persson, "Investigation of brain tissue segmentation error and its effect on EEG source localization," in Proc. Annu. Int. Conf. IEEE Eng. Med. Biol. Soc., Sep. 2012, pp. 1522-1525.

[35] S. M. Smith et al., "Advances in functional and structural MR image analysis and implementation as FSL," NeuroImage, vol. 23, pp. 208-219, 2004.

[36] FreeSurfer [Online]. Available: http://surfer.nmr.mgh.harvard.edu/

[37] C. Wolters, A. Anwander, G. Berti, and U. Hartmann, "Geometry-adapted hexahedral meshes improve accuracy of finite-element-method-based EEG source analysis," IEEE Trans. Biomed. Eng., vol. 54, no. 8, pp. 1446-1453, Aug. 2007.

[38] J. Haueisen, D. S. Tuch, C. Ramon, P. H. Schimpf, V. J. Wedeen, J. S. George, and J. W. Belliveau, "The influence of brain tissue anisotropy on human EEG and MEG," NeuroImage, vol. 15, no. 1, pp. 159-166, 2002. 
[39] K. Foster and H. Schwan, "Dielectric properties of tissues and biological materials: a critical review," Crit. Rev. Biomed. Eng., vol. 17, no. 1, pp. 25-104, 1989.

[40] V. Jurcak, D. Tsuzuki, and I. Dan, “10/20, 10/10, and 10/5 systems revisited: Their validity as relative head-surface-based positioning systems," NeuroImage, vol. 34, no. 4, pp. 1600-1611, 2007.

[41] K. Saladin, Anatomy and Physiology. New York: McGraw-Hill, 2004.

[42] A. Delorme and S. Makeig, "EEGLAB: An open source toolbox for analysis of single-trial EEG dynamics," J. Neurosci. Methods, vol. 134, pp. 9-21, 2004.

[43] W. Penfield and E. Boldrey, "Somatic motor and sensory representation in the cerebral cortex of man as studied by electrical stimulation," Brain, vol. 60, no. 4, pp. 389-443, 1937.

[44] G. Cruccu, M. Aminoff, G. Curio, J. Guerit, R. Kakigi, F. Mauguiere, P. Rossini, R.-D. Treede, and L. Garcia-Larrea, "Recommendations for the clinical use of somatosensory-evoked potentials," Clin. Neurophysiol., vol. 119, no. 8, pp. 1705-1719, 2008 .

[45] J. Spiegel, J. Tintera, J. Gawehn, P. Stoeter, and R. Treede, "Functional MRI of human primary somatosensory and motor cortex during median nerve stimulation," Clin. Neurophysiol., vol. 110, no. 1, pp. 47-52, 1999.

[46] W. Backes, W. Mess, V. van Kranen-Mastenbroek, and J. P. H. Reulen, "Somatosensory cortex responses to median nerve stimulation: fMRI effects of current amplitude and selective attention," Clin. Neurophysiol., vol. 111, no. 10, pp. 1738-1744, 2000.

[47] [Online]. Available: http://www.slicer.org

[48] D. M. Niddam, T. C. Yeh, Y. T. Wu, P. L. Lee, L. T. Ho, L. A. Nielsen, A. C. Chen, and J. C. Hsieh, "Event-related functional MRI study on central representation of acute muscle pain induced by electrical stimulation," NeuroImage, vol. 17, no. 3, pp. 1437-1450, 2002.
[49] K. Torquati, V. Pizzella, S. D. Penna, R. Franciotti, C. Babiloni, P. Rossini, and G. Romani, "Comparison between SI and SII responses as a function of stimulus intensity," J. Neurorep., no. 6, pp. 813-819, 1996.

[50] T. Kawamura, N. Nakasato, K. Seki, A. Kanno, S. Fujita, S. Fujiwara, and T. Yoshimoto, "Neuromagnetic evidence of pre- and post-central cortical sources of somatosensory evoked responses," Clin. Neurophysiol., no. 100, pp. 44-50, 1996.

[51] M. Rullmann, A. Anwander, M. Dannhauer, S. Warfield, F. Duffy, and C. Wolters, "EEG source analysis of epileptiform activity using a 1 $\mathrm{mm}$ anisotropic hexahedra finite element head model," Neuroimage, vol. 44, pp. 399-410, 2009.

[52] C. H. Wolters, A. Anwander, X. Tricoche, D. Weinstein, M. A. Koch, and R. S. MacLeod, "Influence of tissue conductivity anisotropy on EEG/MEG field and return current computation in a realistic head model: A simulation and visualization study using high-resolution finite element modeling," NeuroImage, vol. 30, no. 3, pp. 813-826, 2006.

[53] Q. Mahmood, Y. Shirvany, A. Mehnert, A. Chodorowski, J. Gellermann, F. Edelvik, A. Hedstöm, and M. Persson, "On the fully automatic construction of a realistic head model for EEG source localization," in Proc. Annu. Int. Conf. IEEE Eng. Med. Biol. Soc., Jul. 2013, pp. 3331-3334.

[54] M. Thordstein and R. Constantinescu, "Possibly lifesaving, noninvasive, EEG-guided neuromodulation in anesthesia-refractory partial status epilepticus," Epilepsy Behav., vol. 25, no. 3, pp. 468-472, 2012.

Authors' photographs and biographies not available at the time of publication. 